\title{
Moderate-intensity Treadmill And Ergocycle Exercise Reduce Fasting Blood Glucose Levels In Obese Women
}

\author{
Olivia Andiana ${ }^{a^{*}}$, Slamet Raharjo ${ }^{\mathrm{a}}$, Rias Gesang Kinanti ${ }^{\mathrm{a}}$, Ghana Firsta Yosika ${ }^{\mathrm{b}}$, \\ Ahmad Syahru Mubarok Harisman ${ }^{\mathrm{a}}$ \\ *Corresponding author: Olivia Andiana, E-Mail: olivia.andiana.fik@um.ac.id
}

a. Department of Sport Science, Faculty of Sport Science State University of Malang, Malang, Indonesia

b. Sport Science Study Program, Postgraduate Program, State University of Yogyakarta, Yogyakarta, Indonesia

\begin{abstract}
The study aimed to analyze the decrease in fasting blood glucose levels after moderate-intensity treadmills and ergocycle exercise in obese women. The quasi-experiment method used in this study with two groups pretest-posttest design design. A total of 20 obese women aged 19-23 years participated in the study and were divided into two groups, namely acute ergocycle exercise (AEE, $n=10$ ), and acute treadmill exercise (ATE, $n=10$ ). Exercise is done a single session with an intensity of $60-70 \% \mathrm{HR}_{\max }$ for 40 minutes using ergocycle (Monark $828 \mathrm{E}$ ) and Richter Treadmill (4.0 HP DC). Blood collection is done 30 minutes pre-exercise and 5 minutes post-exercise on capillary blood vessels in the fingerstick. Measurement of fasting blood glucose levels is done using Accu-Chek Performance (Roche, Mannheim, Germany). Data analysis using Independent Samples T-Test with SPSS version 21. The results found that mean of the fasting blood glucose level pre-exercise on AEE $(97.80 \pm 2.20 \mathrm{mg} / \mathrm{dL})$, ATE $(95.60 \pm 3.86 \mathrm{mg} / \mathrm{dL})$, and $(\mathrm{P}>0.05)$. Mean of post-exercise fasting blood glucose levels on AEE $(93.30 \pm 3.09 \mathrm{mg} / \mathrm{dL})$, ATE $(84.60 \pm 6.28 \mathrm{mg} / \mathrm{dL})$, and $(\mathrm{P}<0.001)$. Mean of delta fasting blood glucose levels in AEE $(-4.50 \pm 2.95 \mathrm{mg} / \mathrm{dL})$, ATE $(-11.00 \pm 6.99 \mathrm{mg} / \mathrm{dL})$, and $(\mathrm{P}<0.05)$. Based on the results of research it can be concluded that ergocyle and treadmill exercise with moderate-intensity lowers fasting blood glucose levels. But treadmill exercise is more effective in lowering fasting blood glucose levels than ergocycle exercise. Future research is recommended to compare moderate-intensity treadmill exercise vs. moderate-intensity resistance exercise against decreased fasting blood glucose levels in obese women.
\end{abstract}

Keywoard: Fasing blood glucose levels, treadmill exercise, ergocycle exercise, obesity

\section{Introduction}

Obesity is a health problem that has become an epidemic globally (Carbone et al., 2019). Based on World Health Organization (WHO) (2021), more than 1.9 billion adults over the age of 15 are overweight and 650 million are obese. In Indonesia, prevalence rates of obesity have increased from year to year (Suryadinata \& Sukarno, 2019). Based on Indonesian Basic Health Research (Riskesdas) reported that the prevalence rate of obesity at the age of over 18 years increased from 2007 to $10.6 \%$ and increased to $14.8 \%$ in 2013, and increased again in 2018 to reach $21.8 \%$ (Kemenkes RI, 2018). This is one of the main health problems in the world that must be solved immediately (Seidell \& Halberstadt, 2015).

Obesity is a health problem arising from the complex interaction of genetic, nutritional, and metabolic factors (De Lorenzo et al., 2016). Obesity is not only considered a disease, but obesity is a source of many dangerous diseases, such as hypertension, chronic inflammation, increased oxidative stress, insulin resistance, metabolic glucose disorders (Lasker et al., 2019). All of these metabolic syndromes are closely related to weight gain (Polii et al., 2016). As is the case with metabolic glucose and insulin resistance which directly begins with weight gain (Polii et al., 2016). Obesity is an underlying risk factor for the onset and development of insulin resistance. Among the possible causes of obesity-induced metabolic disorders, adipose organ dysfunction and changes in adipose metabolic processes play a fundamental role (Barazzoni et al., 2018). Obesity can be caused by a long-term imbalance between the total energy that comes in and the total energy expended (Rambhojan et 
al., 2015). The cause of this imbalance between intake and burning of calories is still unclear, but the process of obesity involves several factors such as genetics, environment (lifestyle), and psychology. The impact of insulin resistance and impaired glucose tolerance in obese people will certainly affect blood glucose levels. Blood glucose is a health term that refers to the glucose content in the bloodstream in the body, so it is at risk of prediabetes. Prediabetes is a condition in which blood glucose levels are higher than normal, but not enough to diagnose as diabetes. If not treated properly, prediabetes can develop into diabetes (Sulistiowati, 2018).

Exercise is one of the strategies that can be done to reduce insulin resistance (Amanat, 2020). Exercise can fix glucose levels perfectly, this is evidenced by the decrease in Hemoglobin A1c (HbAlc) concentration which is a benchmark for a reduced risk of diabetes complications. In addition, exercise benefits body fat, arterial blood pressure, vasodilation of endothelium-dependent vessels, blood flow in the skin, the results of comparison between heart rate and blood pressure (both at rest and active) (Lisiswanti \& Cordita, 2016). Muscle contraction can trigger the insertion of Glucose transporter type 4 (GLUT4) into the plasma membrane of active muscle cells. During physical exercise, coordinated increases in the blood flow of skeletal muscles, capillary recruitment, translocation of GLUT-4 to sarcolemma and tubule-T, and metabolism are all essential for glucose absorption and oxidation. Translocation of GLUT-4 to sarcolemma and tubule-T is the basis for skeletal muscle glucose absorption and includes the uptake of GLUT-4 from intracellular storage sites. Glucose absorption during exercise depends on the exercise conditions determined primarily by the intensity and duration of the exercise (Richter \& Hargreaves, 2013). Exercise with ergocycle and treadmill is an exercise that is widely recommended for people with obesity (Nakhanakhup et al., 2006). This is because ergocycle and treadmill exercises can be used to safely combine aerobic motion mechanisms and anaerobic exercise. Exercise with ergocycle or treadmill is safe for obese people who have joint disorders and can protect them from hot weather and crowds. In addition, exercise with ergocycle and treadmill can be controlled exercise intensity according to the ability of patients and researchers. Exercise with ergocycle is also a recommendation as an instrument of measurement of physical exercise research, especially blood pressure measurement during exercise (Willmore \& Costill, 2004). Therefore, it aims to prove the difference in the effect of moderate-intensity treadmill and ergocycle exercise on the decrease in fasting blood glucose levels of obese adolescent women.

\section{Materials and Methods}

The Quasi-experiment was used in this study with two groups pretest-posttest design. A total of 20 obese women participated in the study and were divided into two groups, namely acute ergocycle exercise (AEE, $\mathrm{n}=10$ ), and acute treadmill exercise (ATE, $\mathrm{n}=10$ ). Subjects inclusion criteria, namely the age of 19-23 years, body mass index (BMI) $26-36 \mathrm{~kg} / \mathrm{m}^{2}$, normal blood pressure, normal resting heart rate, and have no history of chronic disease. Subjects sampling uses consecutive sampling techniques. All subjects obtained information through writing and orally about this study. Subjects fill out and sign informed consent before participating in the study. All of these research procedures have been approved by the Health Research Ethics Commission of the Faculty of Medicine, Universitas Brawijaya Malang.

Ergocycle and treadmill exercises are carried out in the Laboratory of the Department of Sports Sciences, Faculty of Sports Sciences, Malang State University. Ergocycle and treadmill exercises are performed at an intensity of $60-70 \% \mathrm{HR}_{\max }$ for 40 minutes with details of 5 minutes of warming up (50-60\% $\mathrm{HR}_{\max }$ ), 30 minutes of the main exercise performed continuously $\left(60-70 \%\right.$ HRmax) and 5 minutes of cooling down $\left(50-60 \% \mathrm{HR}_{\max }\right)$ 
(Rejeki et al., 2021). Ergocycle and treadmill exercises are done at 07.00-09.00 WIB using Ergocycle (Monark 828 E) and Richter Treadmill (4.0 HP DC). Heart rate monitoring during ergocycle exercise and moderateintensity treadmill using Polar Heart Rate Monitor (Polar H10 Heart Rate Sensor, Inc., USA).

Body height measurements using a stadiometer (Portable Seca ${ }^{\circledR}$ Stadiometer, North America). Body weight measurement using digital scales (OMRON Model HN-289, Omron Co., Osaka, Japan). Body mass index (BMI) is measured by calculating weight in kilograms divided by the square of height in meters $\left(\mathrm{kg} / \mathrm{m}^{2}\right)$. Blood pressure measurements using a sphygmomanometer OMRON (OMRON Model HEM-7130 L, Omron Co., Osaka, Japan) on non-dominant arm sections 3 times in a row at intervals of 1-2 minutes between two measurements. At the time of the measurement of the blood pressure, the subjects were in a sitting position. Resting heart rate measured using Beurer Pulse Oximeter (PO 30 Pulse Oximeter). Blood collection is done 30 minutes pre-exercise and 5 minutes post-exercise on capillary blood vessels in the fingerstick. Measurement of fasting blood glucose levels is done using Accu-Chek Performa (Roche, Mannheim, Germany) in milligrams per deciliter ( $\mathrm{mg} / \mathrm{dL})$.

Statistical analysis using SPSS software version 21. The normality test uses the Shapiro-Wilk test, while the other test uses the Paired Sample T-Test and the Independent Samples T-Test. All data is displayed with the mean \pm Standard Deviation (SD). All statistical analyses use significant levels $(\mathrm{P}<0.05)$.

\section{Results}

Statistical analysis of the characteristic data of the study subjects including age, height, weight, body mass index (BMI), systole blood pressure (SBP), diastole blood pressure (DBP), and resting heart rate (RHR) in each group is presented in table 1 below.

Table 1. Results of statistical analysis characteristics of research subjects

\begin{tabular}{|c|c|c|c|c|c|}
\hline \multirow{3}{*}{ Parameters } & \multicolumn{4}{|c|}{ Group } & \multirow{3}{*}{ P-value } \\
\hline & \multicolumn{2}{|c|}{$\operatorname{AEE}(n=10)$} & \multicolumn{2}{|c|}{ ATE $(n=10)$} & \\
\hline & Mean & SD & Mean & SD & \\
\hline Age (years) & 21.00 & 1.33 & 21.10 & 0.88 & 0.845 \\
\hline $\mathrm{BH}(\mathrm{m})$ & 1.59 & 0.05 & 1.58 & 0.04 & 0.963 \\
\hline BW (kg) & 77.04 & 10.80 & 75.79 & 9.94 & 0.791 \\
\hline BMI $\left(\mathrm{kg} / \mathrm{m}^{2}\right)$ & 30.53 & 3.41 & 30.02 & 2.47 & 0.709 \\
\hline $\mathrm{SBP}(\mathrm{mmHg})$ & 111.10 & 5.07 & 111.90 & 3.70 & 0.351 \\
\hline $\mathrm{DBP}(\mathrm{mmHg})$ & 74.50 & 3.57 & 74.30 & 4.06 & 0.692 \\
\hline RHR (bpm) & 73.60 & 3.69 & 76.30 & 8.00 & 0.908 \\
\hline
\end{tabular}

Description: BH: Body height; BW: Body weight; BMI: Body mass index; SBP: Systolic blood pressure; DBP: Diastolic blood pressure; RHR: Resting heart rate; AEE: Acute ergocycle exercise; ATE: Acute treadmill exercise. p-Value is obtained using the Independent Samples T-Test to compare data on the characteristics of research subjects between AEE and ATE. All data is displayed mean \pm SD.

Based on Table 1, the results of the Independent Samples T-Test showed that there was no significant difference in all the characteristic data of the study subjects in each group $(\mathrm{P}>0.05)$. The results of the test of the difference in fasting blood glucose levels between pre-exercise vs. post-exercise on AEE and ATE can be seen in Figure 1. 
AEE

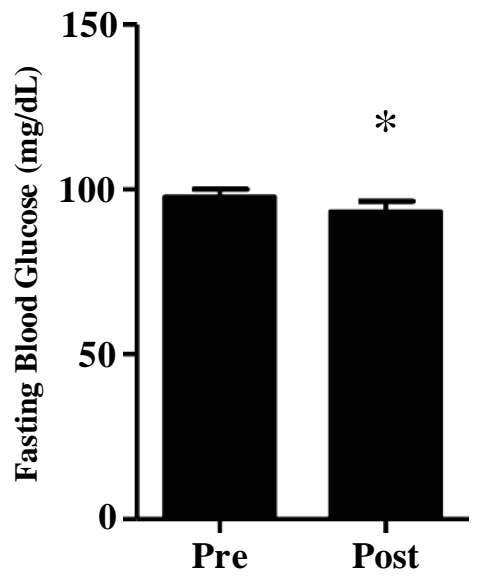

ATE

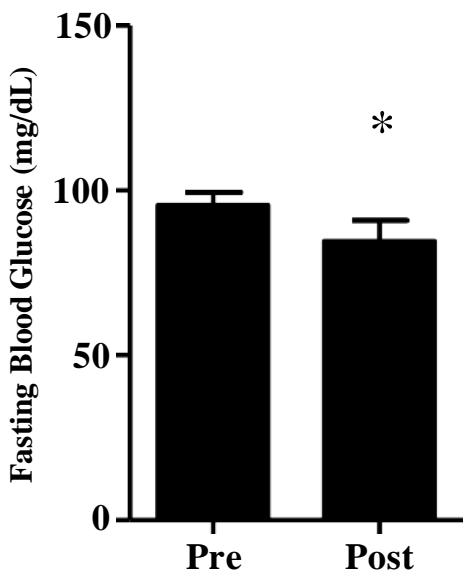

Figure 1. A mean of fasting blood glucose levels in each group

Description: AEE: Acute ergocycle exercise; ATE: Acute treadmill exercise. a p-Value is obtained using the Paired Sample T-Test. All data is displayed mean \pm SD. $(*)$ Significant vs. pre-exercise $(\mathrm{P}<0.001)$

Based on Figure 1 it is seen that there is a decrease in blood glucose levels of fasting post-exercise in each group. The Paired Sample T-Test showed that there was a significant difference in mean of fasting blood glucose levels between pre-exercise vs. post-exercise on AEE and ATE $(\mathrm{P}<0001)$. The results of the analysis of mean of fasting blood glucose levels of pre-exercise (AEE vs. ATE), post-exercise (AEE vs. ATE), and delta (AEE vs. ATE) are presented in Table 2.

Table 2. Fasting pre-exercise blood glucose levels (AEE vs. ATE), post-exercise (AEE vs. ATE), and delta (AEE vs. ATE)

\begin{tabular}{lccccc}
\hline \multirow{2}{*}{ Time } & \multicolumn{3}{c}{ Fasting Blood Glucose $(\mathbf{m g} / \mathbf{d L})$} & & \multirow{2}{*}{ P-value } \\
\cline { 2 - 5 } & \multicolumn{2}{c}{ AEE $(\mathbf{n = 1 0})$} & \multicolumn{2}{c}{ ATE $(\mathbf{n = 1 0})$} & \\
\cline { 2 - 5 } & Mean & SD & Mean & SD & \\
\hline Pre-exercise & 97.80 & 2.20 & 95.60 & 3.86 & 0.140 \\
Post-exercise & 93.30 & 3.09 & 84.60 & 6.28 & $0.001^{*}$ \\
Delta $(\Delta)$ & -4.50 & 2.95 & -11.00 & 6.99 & $0.019^{*}$ \\
\hline
\end{tabular}

Description: AEE: Acute ergocycle exercise; ATE: Acute treadmill exercise. a p-Value is obtained using the Independent Samples T-Test. All data is displayed mean \pm SD. (*) Significant vs. AEE $(\mathrm{P}<0.05)$.

Table 2 showed that mean of pre-exercise fasting blood glucose levels in both groups had the same tendency, while mean of post-exercise fasting blood glucose levels at ATE were lower compared to AEE. Likewise, the mean of delta fasting blood glucose level at ATE was lower compared to AEE. Independent Samples T-Test results showed that there was no significant difference in mean of pre-exercise fasting blood glucose levels between AEE and ATE ( $P>0.05)$, while the mean of post-exercise and delta fasting blood glucose levels between ATE and AEE showed significant differences $(\mathrm{P}<0.05)$.

\section{Discussion}

The study aimed to analyze the difference in the effect of moderate-intensity treadmill and ergocycle exercise on fasting blood glucose levels in obese adolescent women. The main finding in the study was that there was a decrease in the mean of fasting blood glucose levels after a single session of moderate-intensity 
treadmill exercise and ergocycle exercise, but treadmill exercise was more effective in lowering fasting blood glucose levels in obese women than ergocycle exercise. This finding is reinforced by research conducted by Avery \& Walker (2001), reported that a significant lower in blood glucose levels was observed during low- and moderate-intensity exercise compared to rest and this difference was lost 45 minutes after exercise. So did Aslan's research et al. (2019), reported that exercise significantly lowered fasting blood glucose levels. Zheng et al. (2020), also reported that exercise significantly lower blood glucose fluctuations and improved blood glucose control throughout the day. Based on the results of the study, it was shown that lower fasting blood glucose levels are transient. Therefore, in the future, further research is needed on chronic exercise to reduce fasting blood glucose, so that exercise can be applied as a lifestyle in patients who have problems with fasting blood glucose levels.

Based on the results of descriptive analysis, the mean of pre-exercise fasting blood glucose levels showed normal levels in each group. Normal fasting blood glucose levels are between $70 \mathrm{mg} / \mathrm{dL}(3.9 \mathrm{mmol} / \mathrm{L})-100$ $\mathrm{mg} / \mathrm{dL}(5.6 \mathrm{mmol} / \mathrm{L})(\mathrm{CDC}, 2021)$. The results of the one-way ANOVA on pre-exercise showed that there was no significant difference in the mean fasting blood glucose levels between the two groups. This proves that if there are lower fasting blood glucose levels, it may be due to the exercise intervention, not due to differences in the characteristics of the subjects. Several studies show different results in different age groups. Study by Suresh et al. (2017), reported that the age group of 30-60 years showed that obese had high blood glucose levels compared to non-obese. Study by Akter et al. (2017), also reported the same thing, that serum fasting blood glucose increased significantly in obese individuals (aged 25-60 years) in both men and women with levels of $4.07 \pm 0.79 \mathrm{mmol} / \mathrm{l} \& 5.58$, respectively. $\pm 0.64 \mathrm{mmol} / \mathrm{l}$. This is evidence that obesity has a risk of developing type 2 diabetes mellitus (Al-Ghoban \& Khan, 2014).

Diabetes is a chronic disease that occurs either when the pancreas does not produce enough insulin or when the body cannot effectively use the insulin it produces. Insulin is a hormone that regulates blood glucose. Hyperglycemia, or elevated blood glucose, is a common effect of uncontrolled diabetes and over time causes serious damage to many-body systems, especially nerves and blood vessels (WHO, 2021). Fryk (2021), explains that hyperinsulinemia and insulin resistance can occur in obesity, this is a form of homeostatic adaptive response to increased adiposity and free fatty acids (FFA). Reinforced by the statement of Xin et al. (2019), that FFA levels are associated with insulin resistance in non-diabetic subjects, and the Homeostasis Model AssessmentInsulin Resistance (HOMA-IR) in non-obese individuals is more sensitive to changes in FFA. In the future, further research is needed involving the measurement of insulin levels, so that the HOMA-IR can be identified and link obesity with insulin resistance about the risk of diabetes.

\section{Conclusion}

Based on the results of the study, it can be concluded that ergocyle and treadmill exercise with an intensity of $60-70 \% \mathrm{HR}_{\max }$ performed for 30 minutes/exercise session lower fasting blood glucose levels in obese adolescent women. But treadmill exercise is more effective in lowering fasting blood glucose levels than ergocycle exercise. 


\section{Acknowledgments}

We thank the Faculty of Sports Science, the State University of Malang for the 2021 PNBP Research grant No. 6.4.21/UN32.6/LT/2021. Thanks to all participants, research assistants, research colleagues, and laboratory assistants who have been involved in this research.

\section{Reference}

Akter, R., Nessa, A., Husain, M. F., Wahed, F., Khatun, N., Yesmin, M., Nasreen, S., \& Tajkia, T. (2017). Effect of Obesity on Fasting Blood Glucose. Mymensingh medical journal : MMJ, 26(1), 7-11.

Al-Goblan, A. S., Al-Alfi, M. A., \& Khan, M. Z. (2014). Mechanism linking diabetes mellitus and obesity. Diabetes, metabolic syndrome and obesity: targets and therapy, 7, 587-591. https://doi.org/10.2147/DMSO.S67400.

Amanat, S., Sinaei, E., Panji, M., MohammadporHodki, R., Bagheri-Hosseinabadi, Z., Asadimehr, H., Fararouei, M., \& Dianatinasab, A. (2020). A Randomized Controlled Trial on the Effects of 12 Weeks of Aerobic, Resistance, and Combined Exercises Training on the Serum Levels of Nesfatin-1, Irisin-1 and HOMA-IR. Frontiers in physiology, 11, 562895. https://doi.org/10.3389/fphys.2020.562895.

Avery, M. D., \& Walker, A. J. (2001). Acute effect of exercise on blood glucose and insulin levels in women with gestational diabetes. The Journal of maternal-fetal medicine, 10(1), 52-58. https://doi.org/10.1080/714904296.

Aslan, D., Muammer, R., Kurtulmuş, B.A., \& Develi, E. (2019). Effects of The Exercise and Diet Programs on Body Mass Index and Fasting Blood Glucose Level In Overweight and Obese Subjects. International Journal of Physiotherapy, 6(4), 102-105. https://doi.org/10.15621/ijphy/2019/v6i4/185410.

Barazzoni, R., Bischoff, S. C., Boirie, Y., Busetto, L., Cederholm, T., Dicker, D., Toplak, H., Van Gossum, A., Yumuk, V., \& Vettor, R. (2018). Sarcopenic obesity: Time to meet the challenge. Clinical nutrition (Edinburgh, Scotland), 37(6 Pt A), 1787-1793. https://doi.org/10.1016/j.clnu.2018.04.018.

Carbone, S., Del Buono, M. G., Ozemek, C., \& Lavie, C. J. (2019). Obesity, risk of diabetes and role of physical activity, exercise training and cardiorespiratory fitness. Progress in Cardiovascular Diseases, 62(4), 327333. https://doi.org/10.1016/j.pcad.2019.08.004.

Centers for Disease Control and Prevention. (2021, August 10). Diabetes Tests. Retrieved January 14, 2022, from www.cdc.gov: https://www.cdc.gov/diabetes/basics/getting-tested.html.

De Lorenzo, A., Soldati, L., Sarlo, F., Calvani, M., Di Lorenzo, N., \& Di Renzo, L. (2016). New obesity classification criteria as a tool for bariatric surgery indication. World Journal of Gastroenterology, 22(2), 681-703. https://doi.org/10.3748/wjg.v22.i2.681.

Fryk, E., Olausson, J., Mossberg, K., Strindberg, L., Schmelz, M., Brogren, H., Gan, L. M., Piazza, S., Provenzani, A., Becattini, B., Lind, L., Solinas, G., \& Jansson, P. A. (2021). Hyperinsulinemia and insulin resistance in the obese may develop as part of a homeostatic response to elevated free fatty acids: A mechanistic case-control and a population-based cohort study. EBioMedicine, 65, 103264. https://doi.org/10.1016/j.ebiom.2021.103264.

Kementerian Kesehatan Republik Indonesia (Kemenkes RI). (2018). Basic Health Research Results 2018. Ministry of Health RI, 53(9), pp. 1689-1699. 
Lasker, S., Rahman, M. M., Parvez, F., Zamila, M., Miah, P., Nahar, K., Kabir, F., Sharmin, S. B., Subhan, N., Ahsan, G. U., \& Alam, M. A. (2019). High-fat diet-induced metabolic syndrome and oxidative stress in obese rats are ameliorated by yogurt supplementation. Scientific Reports, 9(1), 1-15. https://doi.org/10.1038/s41598-019-56538-0.

Lisiswati, R., Cordita, R. N. (2016). Physical Activity in Lowering Blood Glucose Levels in Type 2 Diabetes Mellitus. Medical Journal of Lampung University, 5 (3). 140-144

Nakhanakhup, C.,P. Moungmee, et al(2006). Regular Physical Exercise in Patients with type II Diabetes Mellitus. European Review of Aging and Physical Activity 3(1).

Polii, R., Engka, J. N. A., \& Sapulete, I. M. (2016). The relationship between sodium levels and blood pressure in adolescents in the District of West Bolangitang, North Bolaang Mongondow Regency. Journal of EBiomedicine, 4(2), 2-7. https://doi.org/10.35790/ebm.4.2.2016.14862.

Rambhojan, C., Bouaziz-Amar, E., Larifla, L., Deloumeaux, J., Clepier, J., Plumasseau, J., Lacorte, J. M., \& Foucan, L. (2015). Ghrelin, adipokines, metabolic factors in relation with weight status in school-children and results of a 1-year lifestyle intervention program. Nutrition and Metabolism, 12(1), 1-10. https://doi.org/10.1186/s12986-015-0039-9.

Rejeki, P.S., Pranoto, A., Prasetya, R.E., \& Sugiharto. (2021). Irisin serum increasing pattern is higher at moderate-intensity continuous exercise than at moderate-intensity interval exercise in obese females. Comparative Exercise Physiology, 17(5), 475 - 484. https://doi.org/10.3920/CEP200050.

Seidell, J. C., \& Halberstadt, J. (2015). The global burden of obesity and the challenges of prevention. Annals of Nutrition and Metabolism, 66(suppl 2), 7-12. https://doi.org/10.1159/000375143.

Sulistiowati, E., \& Sihombing, M. (2018). Development of Type 2 Diabetes Mellitus from Prediabetes in Bogor, West Java. Journal of Health Service Research and Development, 2(1), 59-69. https://doi.org/10.22435/jpppk.v2i1.53.

Suresh, Konakanchi, Babu, Ramesh, Pagadala, Pravallika \& Parvathi,. (2017). Comparative study of blood glucose levels in obese and non-obese individuals. Biomedicine (India). 37. 295-298.

Suryadinata, R. V., \& Sukarno, D. A. (2019). The Effect of Physical Activity on the Risk of Obesity in Adults. The Indonesian Journal of Public Health, 14(1), 104-114. https://doi.org/10.20473/ijph.vl14il.2019.106116.

World Health Organization. (2021, November 10). Diabetes. Retrieved January 14, 2022, from www.who.int: https://www.who.int/news-room/fact-sheets/detail/diabetes.

Wilmore, Jack H., Costill, David L. (2004). Physiology of Sport and Exercise 3rdEd. USA: Human Kinetics

Xin YL, Wang Y, Chi J, Zhu X, Zhao H, Zhao S, Wang Y. Elevated free fatty acid level is associated with insulin-resistant state in nondiabetic Chinese people. Diabetes Metab Syndr Obes. 2019;12:139-147 https://doi.org/10.2147/DMSO.S186505.

Zheng, X., Qi, Y., Bi, L., Shi, W., Zhang, Y., Zhao, D., Hu, S., Li, M., \& Li, Q. (2020). Effects of Exercise on Blood Glucose and Glycemic Variability in Type 2 Diabetic Patients with Dawn Phenomenon. BioMed research international, 2020, 6408724. https://doi.org/10.1155/2020/6408724. 